\title{
The density Turán problem for some 3-uniform unihypercyclic linear hypergraphs. An efficient testing algorithm
}

\author{
Halina Bielak \\ Institute of Mathematics \\ Maria Curie Skłodowska University \\ Pl. M. Curie-Skłodowskiej 5 \\ 20-031 Lublin, Poland \\ Email: hbiel@hektor.umcs.lublin.pl
}

\author{
Kamil Powroźnik \\ Institute of Mathematics \\ Maria Curie Skłodowska University \\ Pl. M. Curie-Skłodowskiej 5 \\ 20-031 Lublin, Poland \\ Email: kamil.pawel.powroznik@gmail.com
}

\begin{abstract}
Let $\mathcal{H}=(V, \mathcal{E})$ be a 3-uniform linear hypergraph with one hypercycle $\mathcal{C}_{3}$. We consider a blow-up hypergraph $\mathcal{B}[\mathcal{H}]$.

We are interested in the following problem. We have to decide whether there exists a blow-up hypergraph $\mathcal{B}[\mathcal{H}]$ of the hypergraph $\mathcal{H}$, with hyperedge densities satisfying special conditions, such that the hypergraph $\mathcal{H}$ appears in a blow-up hypergraph as a transversal. We present an efficient algorithm to decide whether a given set of hyperedge densities ensures the existence of a 3 -uniform linear hypergraph $\mathcal{H}$ with hypercycle $\mathcal{C}_{3}$ in the blow-up hypergraph $\mathcal{B}[\mathcal{H}]$.

Moreover, we state some relations between roots of the multivariate matching polynomial and the inhomogeneous density Turán problem.
\end{abstract}

Index Terms-blow-up hypergraph; density; Turán density problem; unicyclic hypergraph.

\section{INTRODUCTION}

$\mathbf{L}$ ET $\mathcal{H}=(V, \mathcal{E})$ be a simple, connected and finite hypergraph with the vertex set $V$ and hyperedge set $\mathcal{E}$ (see [2]). Turán [13] stated the first results in extremal graph theory. Then many authors extended this subject and formulated similar and new Turán density problems. Many interesting results for some families of simple graphs were published in [1], [6], [7], [9], [11], [12] and [14] obtained.

In this paper we present some algorithm for testing whether a hypergraph with a given set of hyperedge densities is a factor (a transversal) of a blow-up hypergraph for some unihypercyclic hypergraphs. Our algorithm has the time complexity at most $\mathcal{O}\left(n^{2}\right)$, where $n$ is the number of hyperedges of the hypergraph.

Ealier Csikvári and Nagy [8] discovered an interesting algorithm for testing whether a tree with a given set of edge densities is a factor of a blow-up graph. Some generalization of their algorithm is presented in [4]. In this paper we extend this ideas to create a respective algorithm for the family of 3-uniform linear unihypercyclic hypergraphs with a hypercycle $\mathcal{C}_{3}$.

First we define some notions and notations. Other definitions one can find in [2], [5] and [10].
A hypergraph $\mathcal{H}$ is called linear if any two hyperedges intersect in at most one vertex. A hypergraph $\mathcal{H}$ is called $r$ uniform if each hyperedge consists of $r$ vertices.

A subhypergraph $\mathcal{P}_{t}$ of $\mathcal{H}$ is called a linear hyperpath of length $t$ if the hyperedges of $\mathcal{P}_{t}$ can be labelled by $e_{i}, 0 \leq$ $i \leq t-1$ such that the sequence $\left(e_{0}, e_{1}, . ., e_{t-1}\right)$ satisfies the condition: $\left|e_{i} \cap e_{j}\right|=1$ if and only if $|i-j|=1$ and $e_{i} \cap e_{j}=\emptyset$ if and only if $|i-j|>1$, where $e_{i} \in \mathcal{E}(\mathcal{H})$ (see Fig. 1(a)).

A subhypergraph $\mathcal{C}_{t}$ of $\mathcal{H}, t \geq 3$, is called a linear hypercycle of length $t$ if the hyperedges of $\mathcal{C}_{t}$ can be labelled by $e_{i}, 0 \leq i \leq t-1$ such that the sequence $\left(e_{0}, e_{1}, . ., e_{t-1}\right)$ satisfies the condition: $\left|e_{i} \cap e_{j}\right|=1$ if and only if $|i-j|=1$ or $i=0$ and $j=t-1$ and $e_{i} \cap e_{j}=\emptyset, i \neq j$, in the opposite case, where $e_{i} \in \mathcal{E}(\mathcal{H})$ (see Fig. 2).

A 3-uniform linear unihypercyclic hypergraph $\mathcal{H}$ is a connected linear 3 -uniform hypergraph with one hypercycle $\mathcal{C}_{3}$ (see Fig. 3).

The degree of the vertex $v$ in the hypergraph $\mathcal{H}$ is the number of hyperedges containing this vertex. Each vertex of degree 1 in a hypergraph $\mathcal{H}$ is called the leaf. We say that the hypergraph $\mathcal{H}$ is $r$-regular if each vertex of $\mathcal{H}$ has degree $r$. A hyperedge $e \in \mathcal{E}(\mathcal{H})$ is called a pendant hyperedge if it contains exactly one vertex of degree $>1$.

A set $S \subset V(\mathcal{H})$ is called the independent vertex set if the subhypergraph of $\mathcal{H}$ induced by $S$ has empty set of hyperedges. The set $M \subseteq \mathcal{E}(\mathcal{H})$ is called the matching (or independent hyperedge set) in the hypergraph $\mathcal{H}$ if the subhypergraph of $\mathcal{H}$ induced by $M$ is 1-regular.

Let $\mathcal{H}$ be a 3 -uniform linear hypergraph. For each vertex $i \in$ $V(\mathcal{H})$ we associate $a$ cluster $A_{i}$, as a set of new, independent vertices.

For a hypergraph $\mathcal{H}$ we define a blow-up hypergraph $\mathcal{B}[\mathcal{H}]$ of the hypergraph $\mathcal{H}$ as follows. First we replace each vertex $i \in V(\mathcal{H})$ by a cluster $A_{i}$ and next we create some hyperedges between the clusters $A_{i}, A_{j}$ and $A_{k}$ if $\{i, j, k\}$ is a hyperedge in $\mathcal{H}, i, j, k \in V(\mathcal{H})$. Equivalently each hyperedge in $\mathcal{B}[\mathcal{H}]$ has exactly one vertex from the clusters. 
For any three clusters we define $a$ density between them by the following formula

$$
d\left(A_{i}, A_{j}, A_{k}\right)=\frac{e\left(A_{i}, A_{j}, A_{k}\right)}{\left|A_{i}\right|\left|A_{j}\right|\left|A_{k}\right|},
$$

where $e\left(A_{i}, A_{j}, A_{k}\right)$ denotes the number of hyperedges with one element of each of the clusters $A_{i}, A_{j}$ and $A_{k}$.

The hypergraph $\mathcal{H}$ is a transversal of $\mathcal{B}[\mathcal{H}]$ if $\mathcal{H}$ is a subhypergraph of $\mathcal{B}[\mathcal{H}]$ such that we have a homomorphism

$$
\phi: V(\mathcal{H}) \rightarrow V(\mathcal{B}[\mathcal{H}])
$$

for which $\phi(i) \in A_{i}$ for all $i \in V(\mathcal{H})$. Other terminology: $\mathcal{H}$ is a factor of $\mathcal{B}[\mathcal{H}]$ (see Fig. 1(b)).

A hyperedge $e=\{i, j, k\}$ of the hypergraph $\mathcal{H}$ we denote shortly by $e=i j k$.

The homogeneous density Turán problem for 3-uniform linear hypergraphs can be defined as follows. Let us determine the critical hyperedge density, denoted by $d_{\text {crit }}(\mathcal{H})$, which ensures the existence of the subhypergraph $\mathcal{H}$ of $\mathcal{B}[\mathcal{H}]$ as a transversal. Precisely, assume that all hyperedges $e=\{i, j, k\}$ in the hypergraph $\mathcal{H}$ satisfy the condition

$$
d\left(A_{i}, A_{j}, A_{k}\right)>d_{\text {crit }}(\mathcal{H})
$$

where $i, j, k \in V(\mathcal{H})$. Then, no matter how we construct the blow-up hypergraph $\mathcal{B}[\mathcal{H}]$, it contains the hypergraph $\mathcal{H}$ as a transversal. On the other words, for any value $d<d_{\text {crit }}(\mathcal{H})$ there exists a blow-up hypergraph $\mathcal{B}[\mathcal{H}]$ such that

$$
d\left(A_{i}, A_{j}, A_{k}\right)>d
$$

for all hyperedges $i j k \in \mathcal{E}(\mathcal{H})$ which does not contain $\mathcal{H}$ as a transversal.

Moreover, we define the inhomogeneous density Turán problem for 3-uniform linear hypergraphs as follows. Let us assume that for every hyperedge $e \in \mathcal{E}(\mathcal{H})$ a density $\gamma_{e}$ is given. Now our task is to decide if the set of densities $\left\{\gamma_{e}\right\}_{e \in \mathcal{E}(\mathcal{H})}$ ensure the existence of the hypergraph $\mathcal{H}$ as a transversal or we can construct a blow-up hypergraph $\mathcal{B}[\mathcal{H}]$ such that

$$
d\left(A_{i}, A_{j}, A_{k}\right) \geq \gamma_{i j k},
$$

$\{i, j, k\} \in \mathcal{E}(\mathcal{H})$, but it does not induce the hypergraph $\mathcal{H}$ as a transversal.

This two problems has been studied in [8], [12] for simple graphs which are 2-uniform linear hypergraphs. We extend some of those results to 3-uniform linear hypergraphs with the hypercycle $\mathcal{C}_{3}$.

Let us recall the definition of the multivariate matching polynomial of the hypergraph.

Let $\mathcal{H}$ be a hypergraph and let $x_{e}$ be the vector of variables $x_{e}$, for $e \in \mathcal{E}(\mathcal{H})$. We define the multivariate matching polynomial $F_{\mathcal{H}}\left(\underline{x_{e}}, t\right)$ of the hypergraph $\mathcal{H}$ as follows

$$
F_{\mathcal{H}}\left(\underline{x_{e}}, t\right)=\sum_{M \in \mathcal{M}}\left(\prod_{e \in M} x_{e}\right)(-t)^{|M|},
$$

where the summation goes over all matchings of the hypergraph $\mathcal{H}$, including the empty matching (see Example 1 ). (a)
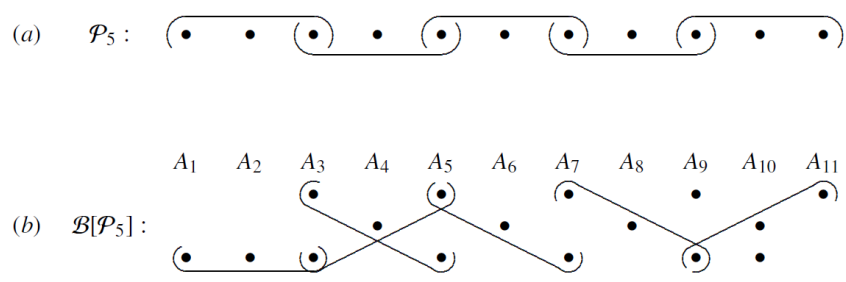

Fig. 1. A 3-uniform linear hyperpath on 5 hyperedges and a blow-up hypergraph $\mathcal{B}\left[\mathcal{P}_{5}\right]$ without the factor $\mathcal{P}_{5}$. Let $\left|A_{i}\right|=1$ for $i \in\{1,2,4,6,8,11\}$ and $\left|A_{i}\right|=2$ for $i \in\{3,5,7,9,10\}$. We obtain the following densities between the clusters in $\mathcal{B}\left[\mathcal{P}_{5}\right]: d\left(A_{1}, A_{2}, A_{3}\right)=d\left(A_{3}, A_{4}, A_{5}\right)=\frac{1}{2}$, $d\left(A_{5}, A_{6}, A_{7}\right)=d\left(A_{7}, A_{8}, A_{9}\right)=d\left(A_{9}, A_{10}, A_{11}\right)=\frac{1}{4}$ and 0 for others. If we add the new hyperedge between clusters $A_{7}, A_{8}, A_{9}$, we get $d\left(A_{7}, A_{8}, A_{9}\right)=\frac{1}{2}$ and $\mathcal{P}_{5}$ as a factor.
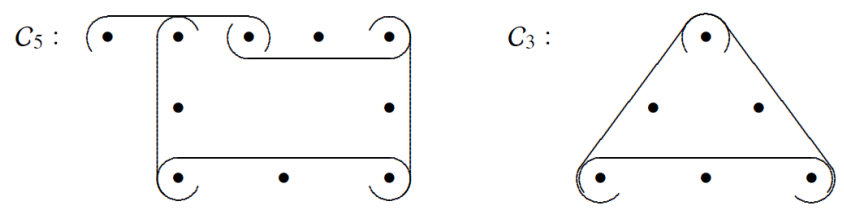

Fig. 2. A 3-uniform linear hypercycles $\mathcal{C}_{5}$ and $\mathcal{C}_{3}$.

The polynomial is the useful tool for the proofs of our results. In particular, we state some relations between roots of the multivariate matching polynomial and the inhomogeneous density Turán problem for 3-uniform linear hypergraphs with the hypercycle $\mathcal{C}_{3}$ which are presented in Theorem 4.

Example 1. Let us consider the 3-uniform linear hypergraph $\mathcal{H}$ with 7 hyperedges as in Fig. 3. Assume that variables $x_{e}$ are given for hyperedges $e \in \mathcal{E}(\mathcal{H})$ as follows

$$
x_{1}=x_{4}=x_{7}=2, x_{2}=x_{6}=1 \text { and } x_{3}=x_{5}=3 .
$$

Then the multivariate matching polynomial of the hypertree $\mathcal{H}$ is presented below

$$
\begin{aligned}
F_{\mathcal{H}}\left(\underline{x_{e}}, t\right) & =1-t\left(x_{1}+x_{2}+x_{3}+x_{4}+x_{5}+x_{6}+x_{7}\right) \\
& +t^{2}\left(x_{1} x_{5}+x_{1} x_{6}+x_{1} x_{7}+x_{2} x_{4}+x_{2} x_{5}\right. \\
& +x_{2} x_{6}+x_{2} x_{7}+x_{3} x_{5}+x_{3} x_{6}+x_{3} x_{7}+ \\
& \left.x_{4} x_{6}+x_{4} x_{7}+x_{6} x_{7}\right)-t^{3}\left(x_{1} x_{6} x_{7}+x_{2} x_{4} x_{6}\right. \\
& \left.+x_{2} x_{4} x_{7}+x_{2} x_{6} x_{7}+x_{3} x_{6} x_{7}+x_{4} x_{6} x_{7}\right) \\
& +t^{4} x_{2} x_{4} x_{6} x_{7}=1-14 t+44 t^{2}-22 t^{3}+4 t^{4} .
\end{aligned}
$$




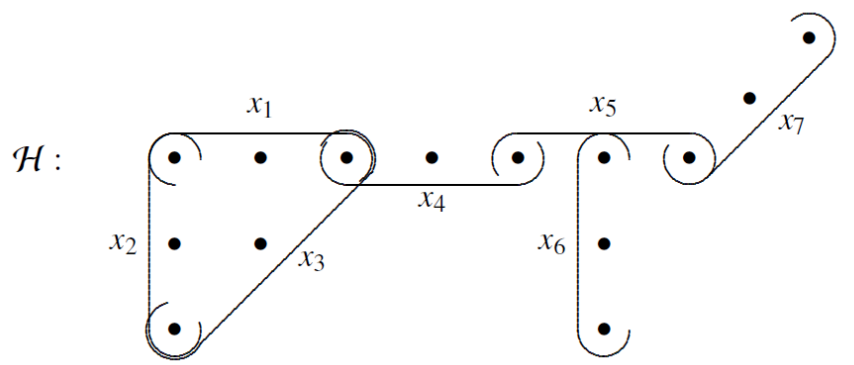

Fig. 3. A 3-uniform linear hypergraph $\mathcal{H}$ with hypercycle $\mathcal{C}_{3}$, where $|V(\mathcal{H})|=14$ and $|\mathcal{E}(\mathcal{H})|=7$, with variables $x_{e}$ assigned to hyperedges $e \in \mathcal{E}(\mathcal{H})$.

\section{THE INHOMOGENEOUS DENSITY TURÁN PROBLEM FOR 3-UNIFORM LINEAR UNIHYPERCYCLIC HYPERGRAPHS WITH HYPERCYCLE $\mathcal{C}_{3}$}

In this section we study the inhomogeneous density Turán problem for 3-uniform linear hypergraphs $\mathcal{H}$ with one hypercycle $\mathcal{C}_{3}$, where a hyperedge density $\gamma_{e}$ is given for each hyperedge $e \in \mathcal{E}(\mathcal{H})$. We extend some results presented in [8], where authors studied the inhomogeneous problem for trees and proved the following theorem.

Theorem 1. (Csikvári, Nagy [8]) Let $T$ be a tree of order $n$ and let $v$ be a leaf of $T$. Assume that for each edge of $T$ a density $\gamma_{e}=1-r_{e}$ is given. Let $T^{\prime}$ be a tree obtained from $T$ by deleting the leaf $v$ and the edge $u v$, where $u$ is the unique neighbour of $v$. Let the edge densities $\gamma_{e}^{\prime}$ in $T^{\prime}$ be defined as follows

$$
\gamma_{e}^{\prime}= \begin{cases}\gamma_{e}=1-r_{e}, & \text { if } e \text { is not incident to } u \text { in } T^{\prime}, \\ 1-\frac{r_{e}}{1-r_{u v}}, & \text { if } e \text { is incident to } u \text { in } T^{\prime} .\end{cases}
$$

Then the set of densities $\left\{\gamma_{e}\right\}_{e \in E(T)}$ ensures the existence of the factor $T$ if and only if all $\gamma_{e}^{\prime} \in(0,1]$ and the set of densities $\left\{\gamma_{e}^{\prime}\right\}_{e \in E\left(T^{\prime}\right)}$ ensures the existence of the factor $T^{\prime}$.

Theorem 1 provides authors of [8] with an efficient algorithm (Algorithm $T$ ) to decide whether a given set of edge densities in a tree ensures the existence of a transversal or does not ensure. Their algorithm is cited on the next page.

We show that their algorithm can be extended for 3 -uniform linear hypergraphs with hypercycle $\mathcal{C}_{3}$. This extension is presented in Algorithm $\mathcal{H C}_{3}$, which is presented in the second half of this paragraph.

Proposition 1. The Algorithm $\mathcal{H C}_{3}$ stops in at most $\mathcal{O}\left(n^{2}\right)$ steps, where $n$ is the number of hyperedges of the input hypergraph.

Proof. Execution time for checking of the property described in Step 0 is at most $\mathcal{O}(n)$, where $n$ is the number of hyperedges of the input hypergraph. Similarly, execution time for checking the first property described in Step 2 is at most $\mathcal{O}(n)$. In the worst case Step 1 is executed at most $\mathcal{O}(n)$, similarly, Step 2, so the time complexity of our algorithm is at most $\mathcal{O}\left(n^{2}\right)$.
The correctness of the Algorithm $\mathcal{H C}_{3}$ follows from the following theorem.

Theorem 2. Let $\mathcal{H}$ be a 3-uniform linear hypergraph with the hypercycle $\mathcal{C}_{3}$. If $|\mathcal{E}(\mathcal{H})|>3$ let $u, v \in V(\mathcal{H})$ be two leaves from a pendant hyperedge $e=\{u, v, w\} \in \mathcal{E}(\mathcal{H})$ for some $w \in V(\mathcal{H})$. Assume that for each hyperedge of $\mathcal{H}$ the density $\gamma_{e}=1-r_{e}$ is given. Let $\mathcal{H}^{\prime}$ be a hypergraph obtained from $\mathcal{H}$ by deleting vertices $u$ and $v$ with the hyperedge uvw. Let the hyperedge densities $\gamma_{e}^{\prime}$ in $\mathcal{H}^{\prime}$ be defined as follows

$$
\gamma_{e}^{\prime}= \begin{cases}\gamma_{e}=1-r_{e}, & \text { if } e \text { is not incident to } w \text { in } \mathcal{H}^{\prime}, \\ 1-\frac{r_{e}}{1-r_{u v w}}, & \text { if } e \text { is incident to } w \text { in } \mathcal{H}^{\prime} .\end{cases}
$$

If $|\mathcal{E}(\mathcal{H})|=3$ (with hyperedge set $\mathcal{E}=\{a y b, a x c, b z c\}$ ), then let $\mathcal{H}^{\prime}$ be a hypertree obtained from $\mathcal{H}$ by deleting a vertex of degree 2, say vertex a, with incident hyperedges ayb and axc. $\mathcal{H}^{\prime}$ is a hyperpath $\mathcal{P}_{b z c}$. Let the density $\gamma_{c z b}^{\prime}$ in $\mathcal{H}^{\prime}$ be defined as follows

$$
\gamma_{b z c}^{\prime}=1-\frac{r_{b z c}}{\left(1-r_{a y b}\right)\left(1-r_{a x c}\right)} .
$$

Then the set of densities $\left\{\gamma_{e}\right\}_{e \in \mathcal{E}(\mathcal{H})}$ ensures the existence of a factor $\mathcal{H}$ if and only if all $\gamma_{e}^{\prime} \in(0,1]$ and the set of densities $\left\{\gamma_{e}^{\prime}\right\}_{e \in \mathcal{E}\left(\mathcal{H}^{\prime}\right)}$ ensures the existence of a factor $\mathcal{H}^{\prime}$.

Proof. Let $\mathcal{H}$ be a 3 -uniform linear hypergraph with one hypercycle $\mathcal{C}_{3}$ and let a density $\gamma_{e}=1-r_{e}$ be given for each $e \in \mathcal{E}(\mathcal{H})$.

$(\Rightarrow)$ First we prove the following statement: if all $\gamma_{e}^{\prime}$ are indeed densities and they ensure the existence of a factor $\mathcal{H}^{\prime}$, then the original densities $\gamma_{e}$ ensure the existence of a factor $\mathcal{H}$.

Let $\mathcal{B}[\mathcal{H}]$ be a blow-up hypergraph of the hypergraph $\mathcal{H}$ such that the density between clusters $A_{i}, A_{j}$ and $A_{k}$ is at least $\gamma_{i j k}$, where $A_{i}, A_{j}, A_{k}$ are clusters of the vertices and $i, j, k \in V(\mathcal{H})$. We show that $\mathcal{B}[\mathcal{H}]$ contains a factor $\mathcal{H}$.

Assume that $|\mathcal{E}(\mathcal{H})|>3$. Let $u, v, w \in V(\mathcal{H})$ and $\{u, v, w\} \in \mathcal{E}(\mathcal{H})$, where $u, v$ are leaves. Define $R_{u, v, w}$ as the subset of $A_{w}$ in the following way (see Fig. 4)

$$
R_{u, v, w}=\left\{x \in A_{w} \mid \exists_{u^{\prime} \in A_{u}, v^{\prime} \in A_{v}}\left\{u^{\prime}, v^{\prime}, x\right\} \in \mathcal{E}(\mathcal{B}[\mathcal{H}])\right\} .
$$

Note that by (1)

$$
\begin{aligned}
\left|R_{u, v, w}\right| \cdot\left|A_{u}\right| \cdot\left|A_{v}\right| & \geq e\left(R_{u, v, w}, A_{u}, A_{v}\right)=e\left(A_{u}, A_{v}, A_{w}\right) \\
& =\gamma_{u v w}\left|A_{u}\right| \cdot\left|A_{v}\right| \cdot\left|A_{w}\right|
\end{aligned}
$$

Hence

$$
\left|R_{u, v, w}\right| \geq \gamma_{u v w}\left|A_{w}\right| \cdot
$$

Now we show the lower bound for the number of hyperedges incident to $R_{u, v, w}$. Let $k, z \in V(\mathcal{H})$ such that $\{k, z, w\} \in$ $\mathcal{E}(\mathcal{H})$. By the inclusion - exclusion formula we count the lower bound for the number of hyperedges between $R_{u, v, w}, A_{k}$ and $A_{z}$ as follows

$$
\begin{gathered}
e\left(R_{u, v, w}, A_{k}, A_{z}\right) \geq \\
e\left(A_{w}, A_{k}, A_{z}\right)-\left(\left|A_{w}\right|-\left|R_{u, v, w}\right|\right) \cdot\left|A_{k}\right| \cdot\left|A_{z}\right|=
\end{gathered}
$$






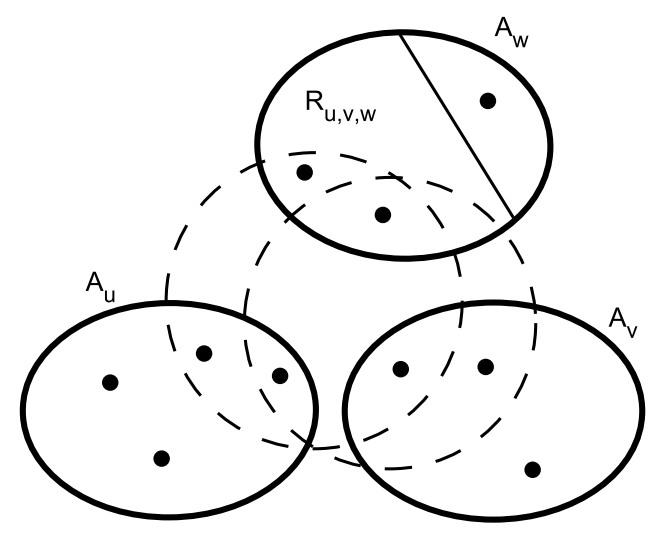

Fig. 4. Clusters $A_{u}, A_{v}$ and $A_{w}$ (bold line) with some hyperedge (broken line) and the set $R_{u, v, w}$

$$
\begin{gathered}
\left|R_{u, v, w}\right| \cdot\left|A_{k}\right| \cdot\left|A_{z}\right|+\left(\gamma_{w k z}-1\right) \cdot\left|A_{w}\right| \cdot\left|A_{k}\right| \cdot\left|A_{z}\right| \geq \\
\left|R_{u, v, w}\right| \cdot\left|A_{k}\right| \cdot\left|A_{z}\right|+\frac{1}{\gamma_{u v w}}\left(\gamma_{w k z}-1\right) \cdot\left|R_{u, v, w}\right| \cdot\left|A_{k}\right| \cdot\left|A_{z}\right|= \\
\left(1-\frac{r_{w k z}}{1-r_{u v w}}\right) \cdot\left|R_{u, v, w}\right| \cdot\left|A_{k}\right| \cdot\left|A_{z}\right|= \\
\gamma_{w k z}^{\prime} \cdot\left|R_{u, v, w}\right| \cdot\left|A_{k}\right| \cdot\left|A_{z}\right| .
\end{gathered}
$$

Now, by deleting the vertex sets $A_{u}, A_{v}$ and $A_{w} \backslash R_{u, v, w}$ from $\mathcal{B}[\mathcal{H}]$, we obtain a hypergraph which is a blow-up hypergraph of $\mathcal{H}^{\prime}$ with the hyperedge densities ensuring the existence of the factor $\mathcal{H}^{\prime}$.

Moreover, by the definition of $R_{u, v, w}$ the factor $\mathcal{H}^{\prime}$ can be extended to a factor $\mathcal{H}$.

Now let us assume that $|\mathcal{E}(\mathcal{H})|=3$, i.e. a hypergraph $\mathcal{H}$ is isomorphic to the hypercycle $\mathcal{C}_{3}$. Let $\mathcal{E}(\mathcal{H})=\{a y b, a x c, b z c\}$, where vertices $a, b, c$ have degree equal to 2 and vertices $x, y, z$ have degree equal to 1 . Let $A_{a}$ be a cluster of the vertex $a$. Define sets $R_{a, y, b}$ and $R_{a, x, c}$ in the following way (see Fig. 5 )

$$
\begin{aligned}
& R_{a, y, b}=\left\{v \in A_{b} \mid \exists_{a^{\prime} \in A_{a}, y^{\prime} \in A_{y}}\left\{a^{\prime}, y^{\prime}, v\right\} \in \mathcal{E}(\mathcal{B}[\mathcal{H}])\right\}, \\
& R_{a, x, c}=\left\{v \in A_{c} \mid \exists_{a^{\prime} \in A_{a}, x^{\prime} \in A_{x}}\left\{a^{\prime}, x^{\prime}, v\right\} \in \mathcal{E}(\mathcal{B}[\mathcal{H}])\right\} .
\end{aligned}
$$

Note that by (1)

$$
\begin{gathered}
\left|R_{a, y, b}\right| \cdot\left|A_{a}\right| \cdot\left|A_{y}\right| \geq e\left(R_{a, y, b}, A_{a}, A_{y}\right)= \\
e\left(A_{b}, A_{a}, A_{y}\right)=\gamma_{a y b}\left|A_{a}\right| \cdot\left|A_{y}\right| \cdot\left|A_{b}\right| \\
\left|R_{a, x, c}\right| \cdot\left|A_{a}\right| \cdot\left|A_{x}\right| \geq e\left(R_{a, x, c}, A_{a}, A_{x}\right)= \\
e\left(A_{c}, A_{a}, A_{x}\right)=\gamma_{a x c}\left|A_{a}\right| \cdot\left|A_{x}\right| \cdot\left|A_{c}\right| .
\end{gathered}
$$

Hence we have the following lower bounds for the cardinalities of $R_{a, y, b}$ and $R_{a, x, c}$

$$
\left|R_{a, y, b}\right| \geq \gamma_{a y b}\left|A_{b}\right|
$$


Algorithm $\mathcal{H C}_{3}$ (for 3-uniform linear hypergraph with a hypercycle $\mathcal{C}_{3}$ )

Input: a 3-uniform linear hypergraph $\mathcal{H}$ with one hypercycle $\mathcal{C}_{3}$ with the set of hyperedge densities $\left\{\gamma_{e}\right\}_{e \in \mathcal{E}(\mathcal{H})}$.

Output: a boolean value

$$
D= \begin{cases}T R U E, & \text { the densities } \gamma_{e} \text { ensure the existence of a factor } \mathcal{H}, \\ F A L S E, & \text { the densities } \gamma_{e} \text { does not ensure the existence of a factor } \mathcal{H} .\end{cases}
$$

Consider a weighted hypergraph $\left(\mathcal{H}, r_{e}\right)$, where $r_{e}=1-\gamma_{e}$.

Step 0 .

if $|E(\mathcal{H})| \geq 1$ and there exists a hyperedge $e \in \mathcal{E}(\mathcal{H})$ for which $r_{e} \geq 1$ then $D:=F A L S E ;$ STOP;

Step 1.

if $|E(\mathcal{H})|=1$ (means $\mathcal{H}$ is a hyperpath $\mathcal{P}_{1}$ ) and $0 \leq r_{e}<1$ then $D:=T R U E ;$ STOP;

Step 2.

if $|E(\mathcal{H})|>3$ then

pick two leaves $u, v$ from a pendant hyperedge $f=\{u, v, w\} \in \mathcal{E}(\mathcal{H})$. Let

$\mathcal{H}^{\prime}=(V(\mathcal{H})-\{u, v\}, \mathcal{E}(\mathcal{H})-\{\{u, v, w\}\})$ and for each hyperedge $e \in \mathcal{E}\left(\mathcal{H}^{\prime}\right)$ set

$$
r_{e}^{\prime}= \begin{cases}r_{e}, & \text { if } e \cap f=\emptyset, \\ \frac{r_{e}}{1-r_{u v w}}, & \text { if } e \cap f=\{w\}\end{cases}
$$

if $|E(\mathcal{H})|=3(\mathcal{E}(\mathcal{H})=\{a y b, a x c, b z c\})$ then

pick vertex of degree equal to 2 , say vertex $a$, and let $\mathcal{H}^{\prime}=(V(\mathcal{H})-\{a, y, x\}, \mathcal{E}(\mathcal{H})-\{a y b, a x c\})$. For hyperedge $e=b z c \in \mathcal{E}\left(\mathcal{H}^{\prime}\right)$ set

$$
r_{e}^{\prime}=r_{b z c}^{\prime}=\frac{r_{b z c}}{\left(1-r_{a y b}\right)\left(1-r_{a x c}\right)}
$$

if $r_{e}^{\prime} \geq 1$ for some hyperedge $e \in \mathcal{E}\left(\mathcal{H}^{\prime}\right)$ then

$D:=F A L S E ;$ STOP;

Go to Step 1 with $\left(\mathcal{H}, r_{e}\right):=\left(\mathcal{H}^{\prime}, r_{e}^{\prime}\right)$.

and

$$
\left|R_{a, x, c}\right| \geq \gamma_{a x c}\left|A_{c}\right| \text {. }
$$

Next let us show how many hyperedges are incident to the sets $R_{a, y, b}$ and $R_{a, x, c}$. By the inclusion - exclusion formula we count the lower bound for the number of hyperedges between $R_{a, y, b}$ and $R_{a, x, c}$

$$
\begin{gathered}
e\left(R_{a, y, b}, R_{a, x, c}, A_{z}\right) \geq e\left(A_{b}, A_{c}, A_{z}\right)- \\
\left(\left|A_{b}\right|-\left|R_{a, y, b}\right|\right) \cdot\left|A_{c}\right| \cdot\left|A_{z}\right|-\left(\left|A_{c}\right|-\left|R_{a, x, c}\right|\right) \cdot\left|A_{b}\right| \cdot\left|A_{z}\right|+ \\
\left(\left|A_{b}\right|-\left|R_{a, y, b}\right|\right)\left(\left|A_{c}\right|-\left|R_{a, x, c}\right|\right) \cdot\left|A_{z}\right|=\left|R_{a, y, b}\right| \cdot\left|R_{a, x, c}\right| \cdot\left|A_{z}\right|+ \\
\left(\gamma_{b c z}-1\right) \cdot\left|A_{b}\right| \cdot\left|A_{c}\right| \cdot\left|A_{z}\right| \geq\left|R_{a, y, b}\right| \cdot\left|R_{a, x, c}\right| \cdot\left|A_{z}\right|+ \\
\left(\gamma_{b c z}-1\right) \frac{1}{\gamma_{a y b}} \frac{1}{\gamma_{a x c}} \cdot\left|R_{a, y, b}\right| \cdot\left|R_{a, x, c}\right| \cdot\left|A_{z}\right|=
\end{gathered}
$$

$$
\begin{gathered}
\left(1-\frac{r_{b z c}}{\left(1-r_{a y b}\right)\left(1-r_{a x c}\right)}\right) \cdot\left|R_{a, y, b}\right| \cdot\left|R_{a, x, c}\right| \cdot\left|A_{z}\right|= \\
\gamma_{b z c}^{\prime}\left|R_{a, y, b}\right| \cdot\left|R_{a, x, c}\right| \cdot\left|A_{z}\right| .
\end{gathered}
$$

Now, by deleting the vertex sets $A_{a}, A_{b} \backslash R_{a, y, b}$ and $A_{c} \backslash R_{a, x, c}$ from $\mathcal{B}[\mathcal{H}]$, we obtain a hypergraph which is a blow-up hypergraph of $\mathcal{C}^{\prime}=\mathcal{P}_{1}$, where $V\left(\mathcal{P}_{1}\right)=\{b, z, c\}$, with the hyperedge density ensuring the existence of the factor $\mathcal{P}_{2}$.

Moreover, by the definition of $R_{a, y, b}$ and $R_{a, x, c}$ the factor $\mathcal{P}_{1}$ can be extended to a factor $\mathcal{C}_{3}$.

$(\Leftarrow)$ Note that if $\gamma_{w k z}^{\prime}<0$ then $\gamma_{w k z}+\gamma_{u v w}<1$. So there exists a construction of blow-up hypergraph which does not induce the linear hyperpath $\mathcal{P}_{2}$ with the consecutive vertices $u, v, w, k, z$ and hyperedges $\{u, v, w\},\{w, k, z\}$, where $i \in A_{i}$ 


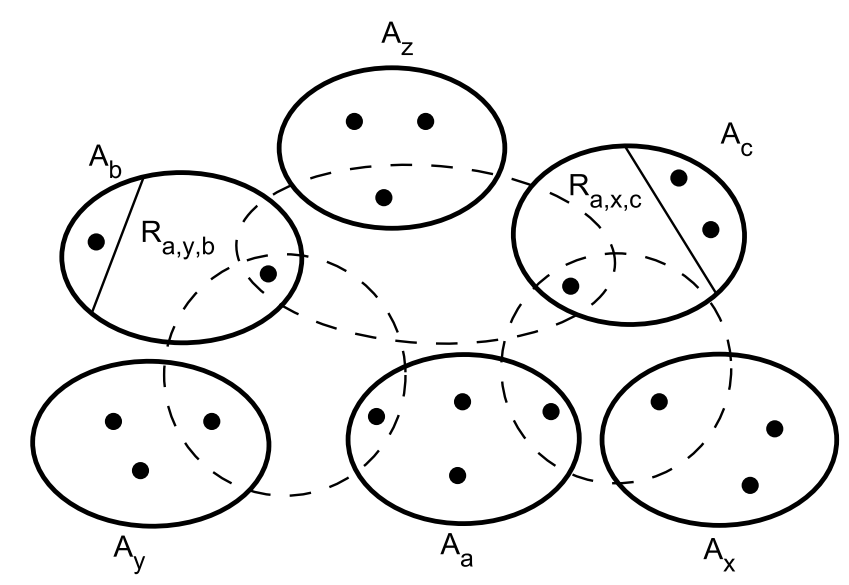

Fig. 5. Clusters $A_{a}, A_{x}, A_{y}, A_{b}$ and $A_{c}$ (bold line) with some hyperedges (broken line) and the sets $R_{a, y, b}$ and $R_{a, x, c}$.



Fig. 6. We assume that $\mathcal{B}^{\prime}\left[\mathcal{H}^{\prime}\right]$ is without a factor $\mathcal{H}^{\prime}$. The construction of the blow-up hypergraph $\mathcal{B}[\mathcal{H}]$ without factor $\mathcal{H}$ for the case where vertices $u, v$ are leaves in $\mathcal{H}$ and $\mathcal{H}^{\prime}=(V(\mathcal{H})-\{u, v\}, \mathcal{E}(\mathcal{H})-\{u v w\})$. The cluster $A_{w}^{\prime}$ is in $\mathcal{B}^{\prime}\left[\mathcal{H}^{\prime}\right]$. Let $A_{w}=\left\{w^{*}\right\} \cup A_{w}^{\prime}, A_{u}=\{u\}$ and $A_{v}=\{v\}$ be clusters in $\mathcal{B}[\mathcal{H}]$. Bold line - cluster, broken line - hyperedge.

for $i \in\{u, v, w, k, z\}$ in this case. Therefore, if some $\gamma_{w k z}^{\prime}<$ 0 then there exists a construction for a blow-up hypergraph of the hypertree $\mathcal{H}$ without a factor $\mathcal{H}$.

Next assume that all the $\gamma_{e}^{\prime}$ are proper densities, but there is a construction of a blow-up hypergraph, say $\mathcal{B}^{\prime}\left[\mathcal{H}^{\prime}\right]$, with hyperedge densities at least $\gamma_{e}^{\prime}$, but which does not induce a factor $\mathcal{H}^{\prime}$. Thus we construct a blow-up hypergraph $\mathcal{B}[\mathcal{H}]$ of the hypertree $\mathcal{H}$ not inducing $\mathcal{H}$. We consider two cases. First, let $|\mathcal{E}(\mathcal{H})|>3$ and $v, u$ be two leaves of $\mathcal{H}$ such that $u v w \in \mathcal{E}(\mathcal{H})$ for some vertex $w \in V(\mathcal{H})$. Let $\mathcal{H}^{\prime}=(V(\mathcal{H})-$ $\{u, v\}, \mathcal{E}(\mathcal{H})-\{u v w\})$. Set $A_{w}=\left\{w^{*}\right\} \cup A_{w}^{\prime}, A_{u}=\{u\}$ and $A_{v}=\{v\}$. We create hyperedges uvw for all $w \in A_{w}^{\prime}$ but do not create $u v w^{*}$ without changing densities in $\mathcal{B}^{\prime}\left[\mathcal{H}^{\prime}\right]$ and with an appropriate density $\gamma_{u v w}$ (see Fig. 6 ).

Now assume that $\mathcal{H}=\mathcal{C}_{3}$ with hyperedge set $\mathcal{E}(\mathcal{H})=$ $\{a y b, a x c, b z c\}$. Let $\mathcal{H}^{\prime}=(V(\mathcal{H})-\{a, y, c\}, \mathcal{E}(\mathcal{H})-$ $\{a y b, a x c\})$, where $a$ is a vertex of $\mathcal{C}_{3}$ of degree 2. Set $A_{b}=\left\{b^{*}\right\} \cup A_{b}^{\prime}, A_{c}=\left\{c^{*}\right\} \cup A_{c}^{\prime}, A_{a}=\{a\}, A_{x}=\{x\}$ and $A_{y}=\{y\}$. We create hyperedges $a y b$ for all $b \in A_{b}^{\prime}$ and $a x c$ for all $c \in A_{c}^{\prime}$ but do not create hyperedges $a y b^{*}$ and $a x c^{*}$ without changing densities in $\mathcal{B}^{\prime}\left[\mathcal{H}^{\prime}\right]$ and with an appropriate

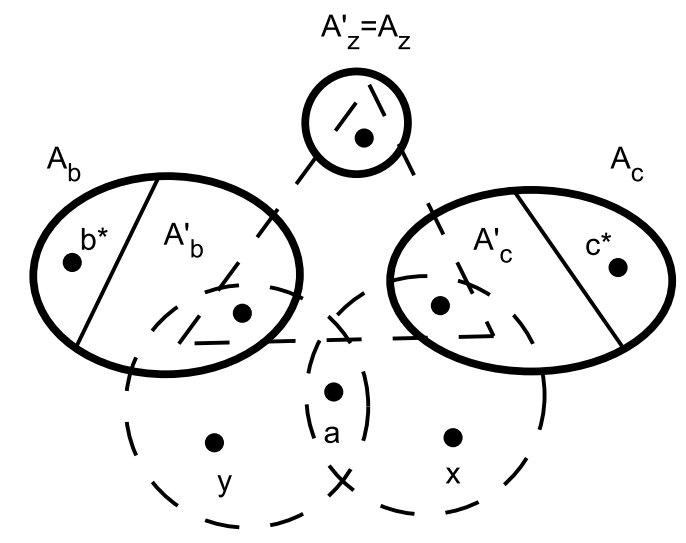

Fig. 7. We assume that $\mathcal{B}^{\prime}\left[\mathcal{H}^{\prime}\right]$ is without a factor $\mathcal{H}^{\prime}$. The construction of the blow-up hypergraph $\mathcal{B}[\mathcal{H}]$ without factor $\mathcal{H}=\mathcal{C}_{3}$ for the case where vertex $a$ has degree 2 in $\mathcal{H}$ and $\mathcal{H}^{\prime}=(V(\mathcal{H})-\{a, x, y\}, \mathcal{E}(\mathcal{H})-\{a y b, a x c\})$. The clusters $A_{b}^{\prime}$ and $A_{c}^{\prime}$ are in $\mathcal{B}^{\prime}\left[\mathcal{H}^{\prime}\right]$. Let $A_{b}=\left\{b^{*}\right\} \cup A_{b}^{\prime}, A_{c}=\left\{c^{*}\right\} \cup A_{c}^{\prime}$, $A_{a}=\{a\}, A_{x}=\{x\}$ and $A_{y}=\{y\}$ be clusters in $\mathcal{B}[\mathcal{H}]$. Bold lines clusters, broken lines - hyperedges.

densities $\gamma_{a y b}$ and $\gamma_{a x c}$ (see Fig. 7).

Example 2. Let us consider a 3-uniform linear hypergraph $\mathcal{H}$ with one hypercycle $\mathcal{C}_{3}$, such that $|\mathcal{E}(\mathcal{H})|=5$ and $|V(\mathcal{H})|=$ 10 , presented in Fig. 8 with two different sets of parameters $\left\{r_{e}\right\}_{e \in \mathcal{E}(\mathcal{H})}$ (in round brackets are given parameters $r_{e}$ from the second set of hyperedge densities). In Table I are presented two different sets of densities $\left\{\gamma_{e}\right\}_{e \in \mathcal{E}(\mathcal{H})}, \gamma_{e}=1-r_{e}$, and changes of parameters $r_{e}$ during the execution of the Algorithm $\mathcal{H C}_{3}$.

We are interested in whether these sets of hyperedge densities ensure an existence of the hypergraph $\mathcal{H}$ as a factor. To solve this problem we use Algorithm $\mathcal{H C}_{3}$. For each hyperedge e a parameter $r_{e}=1-\gamma_{e}$ is assigned as in Fig. 8. Let run Algorithm $\mathcal{H C}_{3}$. All parameters satisfy the condition $0 \leq r_{e}<1$, so we cut the hyperedge $e_{f h i}$ and modify parameters $r_{e}$ by proper formulas presented in the algorithm. We repeat this procedure untill we get a hypergraph with at least one hyperedge $e^{*}$ for which parameter $r_{e^{*}} \geq 1$ or onehyperedge hyperpath (see Fig. 9-12). Notice that we get two different velues at the end. First set of densities $\left\{\gamma_{e}\right\}$ ensure the existence of $\mathcal{H}$ as a factor and the second set $\left\{\gamma_{e}\right\}$ does not ensure.

Now we show some relations between roots of the multivariate matching polynomial and the inhomogeneous density Turán problem. This kind of relations for 3-uniform linear hypertrees have been studied in [4]. Authors of [4] obtained Theorem 3 cited for completeness of this paper. Ealier this subject has been studied by Csikvári and Nagy [8] for trees and in [3] for some family of connected unicyclic graphs.

Theorem 3. (Bielak, Powroźnik [4]) Let $\mathcal{T}=(V, \mathcal{E})$ be a weighted 3-uniform linear hypertree. Let $\gamma_{e}=1-t r_{e}$ be densities assigned to each hyperedge $e \in \mathcal{E}(\mathcal{T})$, where $r_{e} \in$ 
TABLE I

CHANGING OF PARAMETERS $r_{e}$ FOR HYPEREDGES $e \in \mathcal{E}(\mathcal{H})$ DURING THE EXECUTION OF THE ALGORITHM $\mathcal{H} \mathcal{C}_{3}$ FOR TWO DIFFERENT SETS OF HYPEREDGE DENSITIES $\gamma_{e}$ OF THE HYPERGRAPH PRESENTED IN FIG. 8.

\begin{tabular}{|c||c|c|c|c|c|c||c|c|c|c|c|c|}
\hline \multicolumn{1}{|c||}{$e$} & step: & $(1)$ & $(2)$ & $(3)$ & $(4)$ & $(5)$ & step: & $(1)$ & $(2)$ & $(3)$ & $(4)$ & $(5)$ \\
\cline { 2 - 13 } & $\gamma_{e}$ & $r_{e}$ & $r_{e}$ & $r_{e}$ & $r_{e}$ & $r_{e}$ & $\gamma_{e}$ & $r_{e}$ & $r_{e}$ & $r_{e}$ & $r_{e}$ & $r_{e}$ \\
\hline \hline$a b c$ & $3 / 4$ & $1 / 4$ & $1 / 4$ & $1 / 4$ & $10 / 31$ & $40 / 69$ & $1 / 2$ & $1 / 2$ & $1 / 2$ & $1 / 2$ & $7 / 8$ & $175 / 104$ \\
\hline$a x y$ & $3 / 4$ & $1 / 4$ & $1 / 4$ & $1 / 4$ & $1 / 4$ & - & $4 / 5$ & $1 / 5$ & $1 / 5$ & $1 / 5$ & $1 / 5$ & - \\
\hline$y z c$ & $4 / 5$ & $1 / 5$ & $1 / 5$ & $1 / 5$ & $8 / 31$ & - & $4 / 5$ & $1 / 5$ & $1 / 5$ & $1 / 5$ & $7 / 20$ & - \\
\hline$c d e$ & $7 / 8$ & $1 / 8$ & $1 / 8$ & $9 / 40$ & - & - & $3 / 4$ & $1 / 4$ & $1 / 4$ & $3 / 7$ & - & - \\
\hline efg & $2 / 3$ & $1 / 3$ & $4 / 9$ & - & - & - & $2 / 3$ & $1 / 3$ & $5 / 12$ & - & - & - \\
\hline$f h i$ & $3 / 4$ & $1 / 4$ & - & - & - & - & $4 / 5$ & $1 / 5$ & - & - & - & - \\
\hline
\end{tabular}

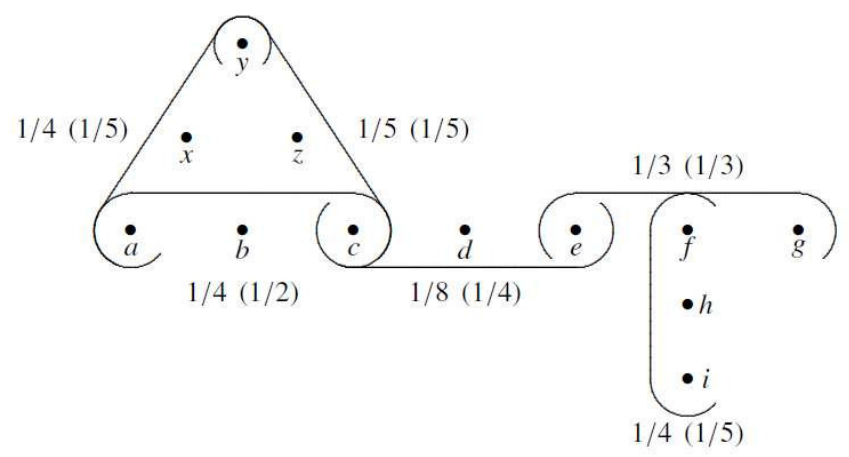

Fig. 8. The input hypergraph $\mathcal{H}$ for Algorithm $\mathcal{H C}_{3}$.

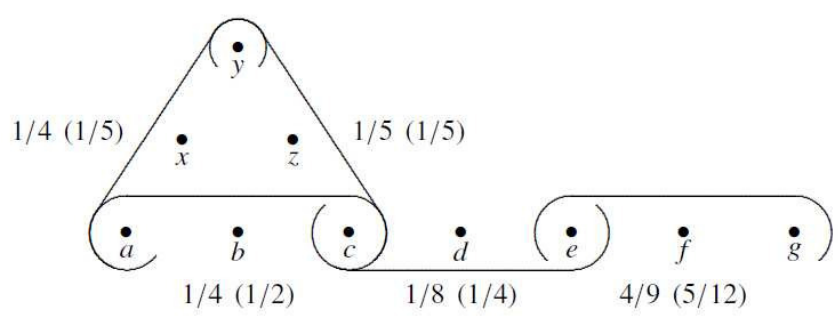

Fig. 9. The hypergraph $\mathcal{H}^{\prime}$ obtained after the first execution of Step 2 of Algorithm $\mathcal{H C}_{3}$ with the hypergraph $\mathcal{H}$ presented in Fig. 8, where the hyperedge $f h i$ was deleted and the parameter $r_{e f g}$ was modyfied according to the first conditional instruction.



Fig. 10. The hypergraph $\mathcal{H}^{\prime}$ obtained after the second execution of Step 2 of Algorithm $\mathcal{H C}_{3}$ with the hypergraph $\mathcal{H}$ presented in Fig. 9, where the hyperedge ef $g$ was deleted and the parameter $r_{c d e}$ was modyfied according to the first conditional instruction.

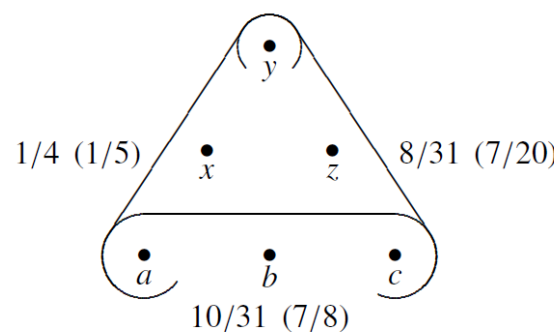

Fig. 11. The hypergraph $\mathcal{H}^{\prime}$ obtained after the third execution of Step 2 of Algorithm $\mathcal{H C}_{3}$ with the hypergraph $\mathcal{H}$ presented in Fig. 10, where the hyperedge $c d e$ was deleted and the parameters $r_{a b c}$ and $r_{y z c}$ were modyfied according to the first conditional instruction. 


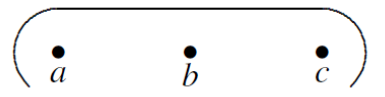

$40 / 69(175 / 104)$

Fig. 12. The hypergraph $\mathcal{H}^{\prime}$ obtained after the last execution of Step 2 of Algorithm $\mathcal{H C}_{3}$ with the hypergraph $\mathcal{H}$ presented in Fig. 11, where the hyperedges $a x y$ and $y z c$ were deleted and the parameter $r_{a b c}$ was modyfied according to the second conditional instruction.

$[0,1)$. Assume that after running of Algorithm $\mathcal{T}$ we get a one-hyperedge hyperpath $\mathcal{P}_{1}$ with

$$
F_{\mathcal{P}_{1}}\left(\underline{r_{e}}, t\right)=0 \text {. }
$$

Then $t$ is a root of the multivariate matching polynomial $F_{\mathcal{T}}\left(\underline{r_{e}}, s\right)$ of the hypertree $\mathcal{T}$.

In Theorem 4 we show similar relation between roots of the multivariate matching polynomial and the inhomogeneous density Turán problem for 3-uniform linear hypergraphs with a hypercycle $\mathcal{C}_{3}$.

Theorem 4. Let $\mathcal{H}=(V, \mathcal{E})$ be a weighted 3-uniform linear unihypercyclic hypergraph with a hypercycle $\mathcal{C}_{3}$. Let $\gamma_{e}=$ $1-t r_{e}$ be densities assigned to each hyperedge $e \in \mathcal{E}(\mathcal{H})$, where $r_{e} \in[0,1)$. Assume that after running of Algorithm $\mathcal{H C}_{3}$ we get a hypercycle $\mathcal{C}_{3}$ with

$$
F_{\mathcal{C}_{3}}\left(\underline{r_{e}}, t\right)=0 .
$$

Then $t$ is a root of the multivariate matching polynomial $F_{\mathcal{H}}\left(\underline{r_{e}}, s\right)$ of the hypergraph $\mathcal{H}$.

Proof. Let $\mathcal{H}=(V, \mathcal{E})$ be a weighted 3 -uniform linear unihypercyclic hypergraph with hypercycle $\mathcal{C}_{3}$. Assume that $|\mathcal{E}(\mathcal{H})|=n$. To prove this theorem we use induction on the number of hyperedges of the hypergraph $\mathcal{H}$.

If this hypergraph consists of 3 hyperedges (i.e., $\mathcal{H}$ is isomorphic to $\mathcal{C}_{3}$, say with $\mathcal{E}(\mathcal{H})=\{a b c, c d e$, ef $a\}$ ), then

$$
F_{\mathcal{H}}\left(\underline{r_{e}}, t\right)=1-t\left(r_{a b c}+r_{c d e}+r_{e f a}\right)
$$

and the condition $F_{\mathcal{H}}\left(\underline{r_{e}}, t\right)=0$ means that $t$ is a root of this multivariate matching polynomial of the hypergraph $\mathcal{H}$.

Assume that the statement of the theorem is true for each hypergraph on at most $n-1$ hyperedges, where $n>3$. Let $\mathcal{H}$ be a hypergraph with $n$ hyperedges and assume that we execute the Algorithm $\mathcal{H C}_{3}$ for a hyperedge $e=\{u, v, w\}$, shortly $u v w$, in the Step 2, where vertices $u, v$ are two leaves in $\mathcal{H}$. Let $\mathcal{H}^{\prime}=\mathcal{H}-\{u, v\}$ be a hypergraph obtained from hypergraph $\mathcal{H}$ by deleting $u$ and $v$ and the hyperedge $u v w$. Densities on hyperedges in hypergraph $\mathcal{H}^{\prime}$ are modyfied by formulas presented in Algorithm $\mathcal{H C}_{3}$. By executing the Algorithm $\mathcal{H C}_{3}$ with input $\mathcal{H}^{\prime}$ we obtain a hypercycle $\mathcal{C}_{3}$ with $F_{\mathcal{C}_{3}}\left(r_{e}^{\prime}, t\right)=0$. By induction we get that $F_{\mathcal{T}^{\prime}}\left(\underline{r_{e}}{ }^{\prime}, t\right)=0$.

Now we apply the formula (2) for hypergraphs $\mathcal{H}^{\prime}$ and $\mathcal{H}$.

We can expand $F_{\mathcal{H}^{\prime}}$ according to whether a monomial contains $x_{w k z}\left(\right.$ where $w k z \in \mathcal{E}\left(\mathcal{H}^{\prime}\right)$ ) or not. Obviously, each monomial contains at most one of the variables $x_{w k z}$ where $w k z \in \mathcal{E}(\mathcal{H})$.

Thus

$$
F_{\mathcal{H}^{\prime}}\left(\underline{x_{e}}, s\right)=Q_{0}\left(\underline{x_{e}}, s\right)-\sum_{\{k, z, w\} \in \mathcal{E}\left(\mathcal{H}^{\prime}\right)} s x_{w k z} Q_{k z}\left(\underline{x_{e}}, s\right),
$$

where $Q_{0}\left(\underline{x}_{e}, s\right)$ consists of those terms which do not contain $x_{w k z}$ and $-s x_{w k z} Q_{k z}\left(\underline{x_{e}}, s\right)$ consists of those terms which contain $x_{w k z}$ (i.e., these terms correspond to the matchings containing the hyperedge $w k z$ ).

We observe that

$$
\begin{gathered}
F_{\mathcal{H}}\left(\underline{x_{e}}, s\right)=\left(1-s x_{u v w}\right) Q_{0}\left(\underline{x_{e}}, s\right)- \\
\sum_{\{k, z, w\} \in \mathcal{E}\left(\mathcal{H}^{\prime}\right)} s x_{w k z} Q_{k z}\left(\underline{x_{e}}, s\right) .
\end{gathered}
$$

Since

$$
\begin{aligned}
& 0=F_{\mathcal{H}^{\prime}}\left(\underline{r_{e}^{\prime}}, t\right)=Q_{0}\left(\underline{r_{e}}, t\right)- \\
& \sum_{\{k, z, w\} \in \mathcal{E}\left(\mathcal{H}^{\prime}\right)} t \frac{r_{w k z}}{1-t r_{u v w}} Q_{k z}\left(\underline{r_{e}}, t\right)
\end{aligned}
$$

we have

$$
\begin{gathered}
\left.0=\left(1-t r_{u v w}\right) F_{\mathcal{H}^{\prime}} \underline{\left(r_{e}^{\prime}\right.}, t\right)=\left(1-t r_{u v w}\right) Q_{0}\left(\underline{r_{e}}, t\right) \\
-\sum_{\{k, z, w\} \in \mathcal{E}\left(\mathcal{H}^{\prime}\right)} \operatorname{tr}_{w k z} Q_{k z}\left(\underline{r_{e}}, t\right)=F_{\mathcal{H}}\left(\underline{r_{e}}, t\right) .
\end{gathered}
$$

So $t$ is a root of $F_{\mathcal{H}}\left(\underline{r_{e}}, s\right)$. The proof is done.

To search more relations between roots of the multivariate matching polynomial and the inhomogeneous density Turán problem for 3-uniform linear hypergraphs with a hypercycle $\mathcal{C}_{3}$ we need analogue result to Lemma 1 presented below. According to our knowledge similar result for hypercycle $\mathcal{C}_{3}$ is not known.

Lemma 1. (Bondy, et al. [6]) Let $\alpha, \beta, \gamma$ be the edge densities between the clusters of a blow-up graph of the triangle - a cycle $C_{3}$. If

$$
\alpha \beta+\gamma>1, \beta \gamma+\alpha>1, \gamma \alpha+\beta>1
$$

then the blow-up graph contains a triangle as a transversal.

\section{CONCLUSION}

In this paper we showed some results for the inhomogeneous density Turán problem of 3 -uniform connected linear unihypercyclic hypergraphs with a hypercycle $\mathcal{C}_{3}$.

We presented Algorithm $\mathcal{H C}_{3}$ for testing whether the 3uniform connected linear hypergraph $\mathcal{H}$ with a hypercycle $\mathcal{C}_{3}$ with a given set of hyperedge densities $\left\{\gamma_{e}\right\}_{e \in \mathcal{E}(\mathcal{H})}$ is a transversal of a blow-up hypergraph $\mathcal{B}[\mathcal{H}]$. The Algorithm $\mathcal{H C}_{3}$ has $\mathcal{O}\left(n^{2}\right)$ time complexity in the worst case, where $n$ is the number of hyperedges of $\mathcal{H}$. In this way we have the answer whether the hyperedge densities ensure the existence of the transversal or does not ensure.

Moreover, we stated Theorem 2 to prove the correctness of the Algorithm $\mathcal{H C}_{3}$. 
Additionally, in Theorem 4, we stated some relation between roots of the multivariate matching polynomial and the inhomogeneous density Turán problem for 3-uniform linear hypergraphs with a hypercycle $\mathcal{C}_{3}$.

Open problem: In the future work we want to find relations between location of roots of the multivariate matching polynomial and the inhomogeneous density Turán problem for $r$-uniform linear hypergraphs with one hypercycle of length $t$, where $t \geq 3$. This problem for trees was studied in [8] and for some connected unicyclic graphs was studied in [3].

\section{REFERENCES}

[1] R. Baber, J.R. Johnson and J. Talbot, The minimal density of triangles in tripartite graphs, LMS J. Comput. Math., 13 (2010), 388-413, http://dx.doi.org/10.1112/S1461157009000436.

[2] C. Berge, Graphs and hypergraphs, Elsevier, New York, NY, USA (1973).

[3] H. Bielak, K. Powroźnik, An efficient algorithm for the density Turán problem of some unicyclic graphs, Annals of Computer Science and Information Systems, Proceedings of the 2014 FedCSIS, Vol. 2 (2014), 479-486, http://dx.doi.org/10.15439/978-83-60810-58-3.
[4] H. Bielak, K. Powroźnik, An efficient algorithm for the density Turán problem of 3-uniform linear hypertrees, unpublished.

[5] B. Bollobás, Extremal Graph Theory, Academic Press (1978).

[6] A. Bondy, J. Shen, S. Thomassé and C. Thomassen, Density Conditions for triangles in multipartite graphs, Combinatorica, 26 (2006), http://dx.doi.org/10.1007/s00493-006-0009-y.

[7] W.G Brown, P. Erdös and M. Simonovits, Extremal problems for directed graphs, Journal of Combinatorial Theory, Series B 15 (1) (1973), 77-93, http://dx.doi.org/10.1016/0095-8956(73)90034-8.

[8] P. Csikvári and Z. L. Nagy, The density Turán Problem, Combinatorics, Probability and Computing, 21 (2012), 531-553, http://dx.doi.org/10.1017/S0963548312000016.

[9] Z. Füredi, Turán type problems, Survey in Combinatorics Vol. 166 of London Math. Soc. Lecture Notes (A.D. Keedwell, ed.) (1991), 253-300, http://dx.doi.org/10.1017/cbo9780511666216.010.

[10] C.D. Godsil and G. Royle, Algebraic Graph Theory, Springer (2001), http://dx.doi.org/10.1007/978-1-4613-0163-9.

[11] G. Jin, Complete subgraphs of $r$-partite graphs, Combin. Probab. Comput., 1 (1992), 241-250, http://dx.doi.org/10.1017/s0963548300000274.

[12] Z.L. Nagy, A multipartite version of the Turán problem - density conditions and eigenvalues, The Electronic Journal of Combinatorics, 18 (2011), \# P46.

[13] P. Turán, On an extremal problem in graph theory, Mat. Fiz. Lapok, 48 (1941), 436-452.

[14] R. Yuster, Independent transversal in $r$-partite graphs, Discrete Math., 176 (1997), 255-261, http://dx.doi.org/10.1016/s0012-365x(96)00300-7. 